\title{
Fission Research at IRMM
}

\author{
F.-J. Hambsch ${ }^{1}$,a , S. Oberstedt ${ }^{1}$, S. Zeynalov ${ }^{1,2}$, N. Kornilov ${ }^{3}$, I. Fabry ${ }^{1}$, R. Borcea ${ }^{1}$, and A. Al-Adili ${ }^{1}$ \\ 1 European Commission JRC-IRMM, Retieseweg 111, B-2440 Geel, Belgium \\ 2 JINR Dubna, Moscow Region, Russia \\ 3 Clippinger Labs, Physics and Astronomy, Ohio University, Athens, OH 45701, USA
}

\begin{abstract}
Fission Research at JRC-IRMM has a longstanding tradition. The present paper is discussing recent investigations of fission fragment properties of ${ }^{238} \mathrm{U}(\mathrm{n}, \mathrm{f}),{ }^{234} \mathrm{U}(\mathrm{n}, \mathrm{f})$, prompt neutron emission in fission of ${ }^{252} \mathrm{Cf}(\mathrm{SF})$ as well as the prompt fission neutron spectrum of ${ }^{235} \mathrm{U}(\mathrm{n}, \mathrm{f})$ and is presenting the most important results.
\end{abstract}

\section{Introduction}

The fission process plays an important role in many applications in nuclear science as well as in basic nuclear physics. Despite the fact that fission is known for more than 70 years the present description of the fission process remains largely dominated by phenomenology. Hence, consistent models to describe the fission process, i. e. the properties of the fissioning system, fission dynamics and fragment distributions, are still lacking. To this end it is important to investigate the fission process from the experimental side in-depth to provide the necessary input parameters for the improvement of nuclear fission models.

\section{Experiments}

The JRC-IRMM has a longstanding tradition in the field of neutron-induced fission physics studies. It is especially well equipped with world-class facilities as the high resolution neutron time-of-flight spectrometer GELINA and the 7MV Van de Graaff accelerator for quasi-monoenergetic neutron production MONNET. During the recent past several neutron induced fission reactions have been studied in the energy range up to 6 $\mathrm{MeV}$ and spontaneous fission. Angular, mass and total kinetic energy (TKE) distributions have been studied for ${ }^{234,238} \mathrm{U}(\mathrm{n}, \mathrm{f})$, prompt neutron emission in spontaneous fission of ${ }^{252} \mathrm{Cf}$ as well as the prompt fission neutron spectrum of ${ }^{235} \mathrm{U}(\mathrm{n}, \mathrm{f})$. In the following chapters the individual isotopes will be discussed and, due to space limitations, only the most important results will be presented.

\footnotetext{
${ }^{a}$ e-mail: Franz-Josef.Hambsch@ec.europa.eu
}

\subsection{Results for ${ }^{234,238} \mathbf{U}(\mathbf{n}, \mathbf{f})$}

Fission fragment properties like mass, angular distribution and TKE have been studied by means of the double-energy (2E) method. As fission fragment detector a twin Frisch-grid ionisation chamber was used. The neutron beam was supplied by the JRCIRMM Van de Graaff accelerator using the ${ }^{3} \mathrm{H}(\mathrm{p}, \mathrm{n})$ and ${ }^{7} \operatorname{Li}(\mathrm{p}, \mathrm{n})$ reaction. More details about the experiment set-up is given in Ref. [1].

The investigations were motivated by recent model calculations, where the concept of multi-modal fission was included into the statistical model to describe neutron-induced cross sections [2]. In particular the outer fission barrier is fragmented into two different asymmetric and one symmetric barrier with the aim to calculate quantitatively fission-fragment data (see Fig. 1). The corresponding barrier parameters were adjusted to reproduce previous experimental fissionfragment data for the case of ${ }^{238} \mathrm{U}(\mathrm{n}, \mathrm{f})[3]$.

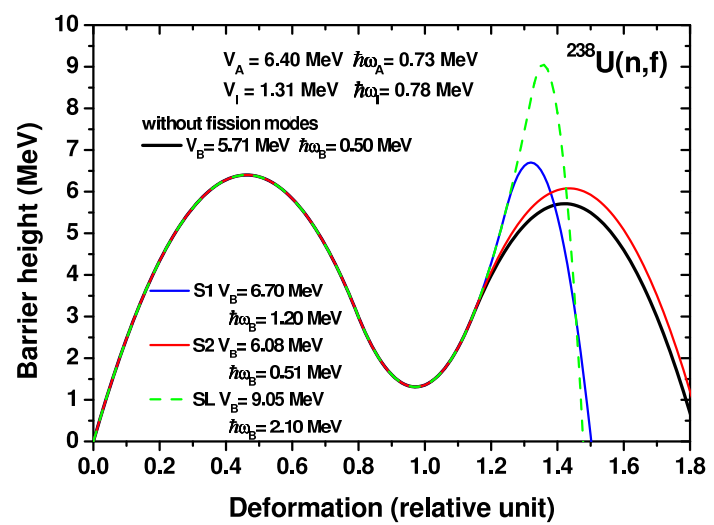

Fig. 1. Fission mode separated fission barriers in ${ }^{239} \mathrm{U}$ with barrier parameters.

This is an Open Access article distributed under the terms of the Creative Commons Attribution-Noncommercial License, which permits unrestricted use, distribution, and reproduction in any noncommercial medium, provided the original work is properly cited. 


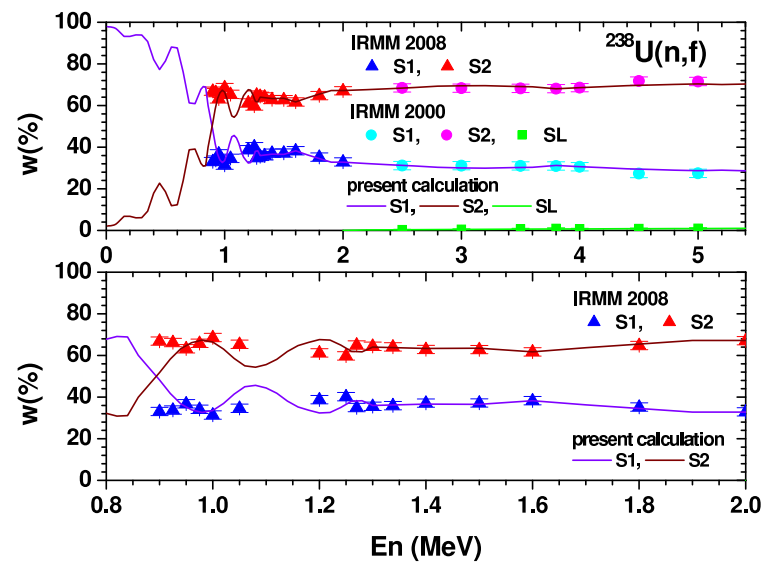

Fig. 2. Modal weights for the standard modes for ${ }^{239} \mathrm{U}$ as a function of incident neutron energy.

This model predicted that in the vibrational resonances at fission threshold of the reaction ${ }^{238} \mathrm{U}(\mathrm{n}, \mathrm{f})$ the charactersitics of the fission fragments show strong changes compared to that observed in the plateau of the cross-section. Consequently, the present experiment covered the incident neutron energy range from $0.9 \mathrm{MeV}$ to $2 \mathrm{MeV}$ in detail. After careful data analysis the expected fluctuations in mean mass and TKE due to a considerable change of the different fission mode weights around the vibrational resonance could not be observed (for details see Ref. [1]). Only the angular distribution showed significant fluctuations for the vibrational resonances (at about $0.9 \mathrm{MeV}$ and 1.2 $\mathrm{MeV}$ ) observed in the threshold of the fission cross section, as well as a broad structure around $1.6 \mathrm{MeV}$ incident neutron energy (see Fig. 3).

Based on the new experimental results the model calculations have been revisited and an improved representation of the barrier parameters (see Fig. 1) as well as a new calculation of the mode weights have been performed (see Fig. 2). The weights of the socalled Standard fission modes as a function of incident neutron energy are shown. The lines are the pedictions

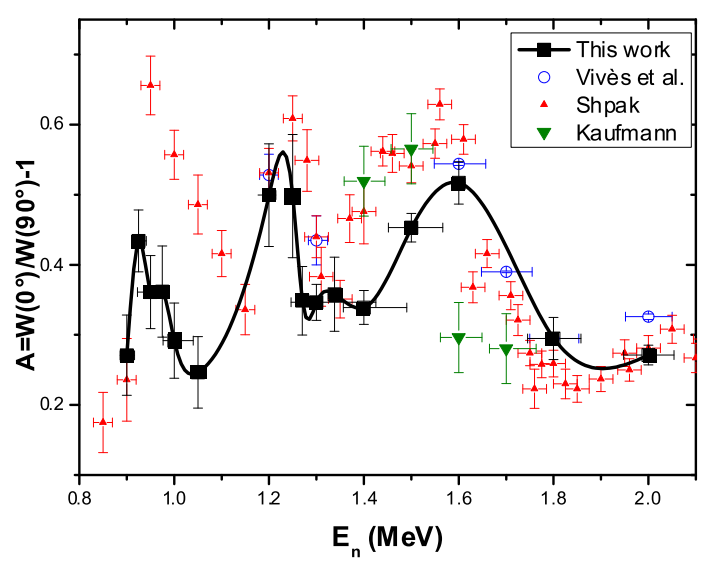

Fig. 3. Anisotropy of the fission fragment angular distribution for ${ }^{238} \mathrm{U}(\mathrm{n}, \mathrm{f})$ as a function of incident neutron energy.

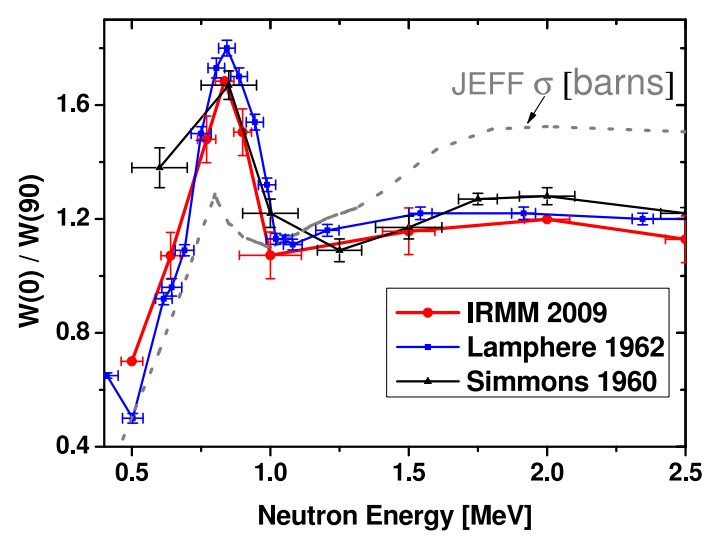

Fig. 4. Anisotropy of the fission fragment angular distribution for ${ }^{234} \mathrm{U}(\mathrm{n}, \mathrm{f})$ as a function of incident neutron energy.

of the model (cf. Ref. [2]) compared to the (recent) experimental findings (full triangles). In the figure already the improved calculation of the mode weights as a function of the incident neutron energy taking our news fission fragment data into account is shown. Possible fluctuations in the fission-fragment characteristics are now shifted to lower incident neutron energy, however, not accessible to present experimental methods. The result shows the still persisting difficulty to make quantitative predictions of fission-fragment data, which might be explained by our limited picture of fission barriers and their simplified parabolic representation.

The experiment ${ }^{234} \mathrm{U}(\mathrm{n}, \mathrm{f})$ has just been started and as a first result the very strong angular anisotropy observed for this nucleus in the vicinity of the vibrational resonance in the threshold of the fission cross section has been verified (see Fig. 4). The measurements are made relative to the neutron induced fission of ${ }^{235} \mathrm{U}$ at thermal energy. Recommended values for TKE and mean heavy mass are taken from Ref. [4]. Possible fluctuations in the other fission fragment

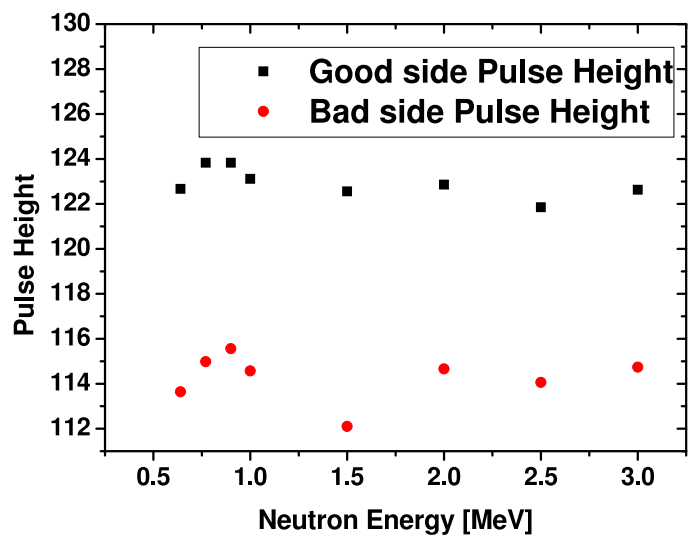

Fig. 5. Average pulse height of the fission fragment distribution for ${ }^{234} \mathrm{U}(\mathrm{n}, \mathrm{f})$ as a function of incident neutron energy. 


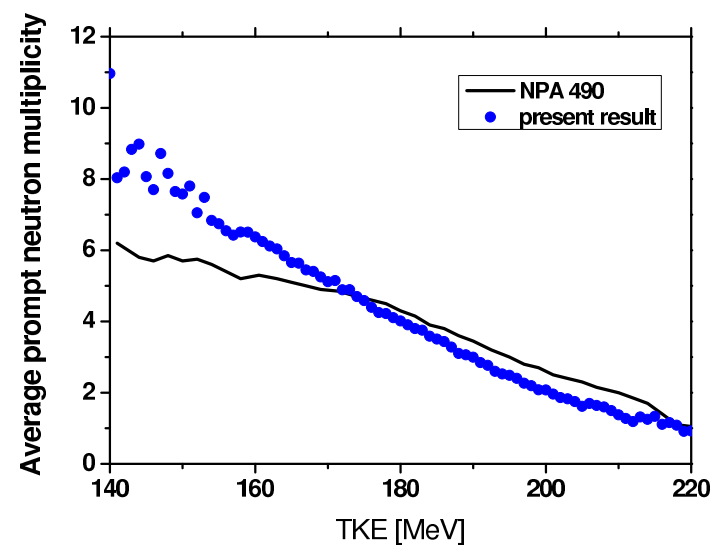

Fig. 6. Prompt neutron multiplicity as a function of fission fragment TKE. The present results (full line) are compared to results from Ref. [5].

properties in the vicinity of the vibrational resonance are visible after a very preliminary anaylysis of the pulse height distributions has been made. Fig. 5 shows the average pulse height of the fission fragments as a function of incident neurton energy. A significant increase in pulse height is observed around $0.8 \mathrm{MeV}$, the position of the vibrational resonance. Further analysis of the data to obtain pre-neutron mass distributions is in progress.

\subsection{Prompt neutron emission in fission of ${ }^{252} \mathrm{Cf}(\mathrm{SF})$}

The pioneering experiment of Budtz-Jorgensen and Knitter [5] was repeated to verify the results with improved statistics using for the first time a digital data acquisition system for the detection of both the fission fragments and prompt neutrons. A twin Frischgrid ionisation chamber was used together with an NE213 equivalent neutron detector to investigate the prompt neutron emission as a function of fragment mass and total kinetic energy. The data analysis is still in progress but first results show the complete absence of a reduction of the prompt neutron multiplicity at low TKE and a different slope of this function towards high TKE (see Fig. 6). This is much in line with physics expectations, because based on energy balance the more neutrons will be emitted the more excitation energy is available at low TKE. A recent calculation based on the Point by Point model of A. Tudora of the same quantity based on experimental mass and TKE distributions from JRC-IRMM revelead the same TKE dependence as given in Fig. 6 [6]. The slope of the distribution $\mathrm{d}(\nu) / \mathrm{d}(\mathrm{TKE})=$ 8.7 MeV. This is also in very good agreement to the excitation energy needed to emit one neutron as mentioned in Ref. [7]. Also the dependence of the prompt neutron multiplicity as a function of mass number A is slightly different in the present study compared to Ref. [5] (see Fig. 7). A more detailed analysis of the

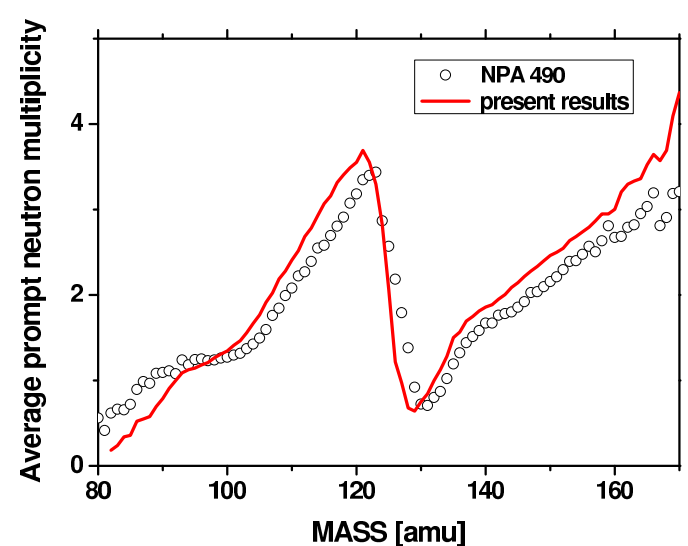

Fig. 7. Prompt neutron multiplicity as a function of fission fragment mass. The present results (full line) are compared to results from Ref. [5].

prompt neutron multiplicity as a function of the fission fragment mass number is in progress. So far, it seems already obvious that a problem exists for this very important data for reactor applications in the literature (e.g. Ref. [5]), which should be further investigated in the near future.

\subsection{Prompt fission neutron spectrum of ${ }^{235} U(n, f)$}

The prompt fission neutron spectrum (PFNS) has been measured at the cold-neutron beam of the Budapest Research Reactor [9]. The experiment was supported within the FP7 project EFNUDAT (European Facilities for Nuclear Data Measurements).

The new experimental data are also compared to literature values of Starostov et al. [11]. A perfect agreement over the full energy range covered in the present study is observed. Three NE213-equivalent neutron scintillation detectors surrounded by large lead and lithium-carbonated paraffine shieldings have been used

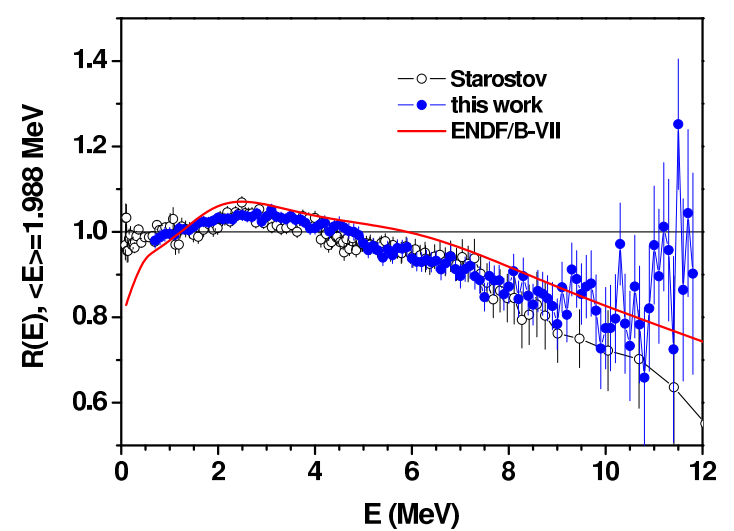

Fig. 8. Ratio of the PFNS for ${ }^{235} \mathrm{U}(\mathrm{n}, \mathrm{f})$ to a Maxwellian disribution with $\mathrm{T}=1.988 \mathrm{MeV}$. The new experimental points (full symbols) are compared to Ref. [11] and to the evaluation based on the Madland-Nix model [10]. 


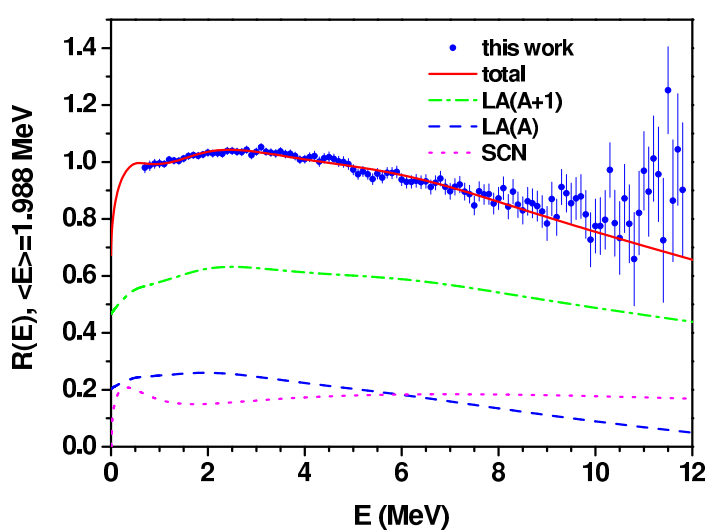

Fig. 9. Inclusion of scission neutron emission based on details given in Ref. [9].

simultaneously together with a double Frisch-grid ionisation chamber for the fission fragment detection. The detailed characterisaton of the detectors and a description of the experiment is given in Ref. $[8,9]$. Here only the resulting PFNS is presented in Fig. 8 relative to a Maxwellian distribution with mean energy $\mathrm{E}=1.988$ $\mathrm{MeV}$. It is obvious that the measured spectrum (symbols) does not agree with the calculation within the so-called Los Alamos model ([10], full line).

To be able to reproduce the measured PFNS, an additional neutron source, the so-called scission neutrons need to be taken into account. Details are given in Ref. [9]. Fig. 9 shows the resulting calculation in comparison with the new experimental data, which show perfect agreement in the full energy range covered. The obvious discrepancy between prompt neutron spectrum model and experimental results triggered an IAEA co-ordinated research project on PFNS going to be organised in the coming years to improve the understanding of this important physical quantity.

\section{Conclusion}

Several fission reactions have been study in the past years. For ${ }^{238} \mathrm{U}(\mathrm{n}, \mathrm{f})$ the theoretically predicted fluctuations in the vibrational resonances in the fission cross section threshold could not be verified experimentally. The input parameters for the statistical model have been revised and adapted to the new results for ${ }^{238} \mathrm{U}(\mathrm{n}, \mathrm{f})$.

For ${ }^{234} \mathrm{U}(\mathrm{n}, \mathrm{f})$ experiments have just started and as a first result the strong angular anisotropy of fission fragment emission could be verified.

In the spontaneous fission of ${ }^{252} \mathrm{Cf}$ for the first time no reduction of the prompt neutron multiplicity as a function of TKE is observed, which is in agreement with the conservation law of energy.

For the PFNS of ${ }^{235} \mathrm{U}(\mathrm{n}, \mathrm{f})$ new experimental results have shown that the present theoretical description of the PFNS in terms of the so-called Los Alamos model [10] is not able to reproduce the experimental data.

\section{References}

1. E. Birgersson et al., Nucl. Phys. A817, (2009) 1-34.

2. F.-J. Hambsch et al., Ann. Nucl. Energy 32, (2005) 1297-1304.

3. F. Vives et al., Nucl. Phys. A662, (2000) 63-92.

4. C. Wagemans, The Nuclear Fission Process (CRC Press, Boca Raton 1991) 323.

5. C. Budtz-Jorgensen and H.-H. Knitter, Nucl. Phys. A490, (1988) 307-328.

6. A. Tudora, private communication (2009).

7. G. Barreau et al., Nucl. Phys. A432, (1985) 411.

8. N.V. Kornilov, I. Fabry, S. Oberstedt, F.-J. Hambsch, Nucl. Inst. Meth. A599 (2009) 226.

9. N. Kornilov et al., Nucl. Sci. Eng. in press.

10. D. Madland and R. Nix, Nucl. Sci. Eng. 81, (1982) 213.

11. B. I. Starastov et al., Nejtronnaja fizika (6-th Conf. for Neutron Phys., Kiev, 1983), 1984, v2, p 285, 290, 294, EXFOR 40871, 40872, 40873. 